\title{
Roman Open Data: una herramienta para la formación del alumnado universitario Roman Open Data: a tool for the academic training of university students
}

\author{
Jordi Pérez González ${ }^{1}$, Juan Manuel Bermúdez Lorenzo ${ }^{1}$, Antonio Aguilera Martín ${ }^{2}$ \\ jordi.perezgonzalez@udg.edu, juan.bermudez@urjc.es, antonioaguilera@ub.edu
}

\author{
${ }^{1}$ Dept. Història \\ i Història de l'Art \\ Universitat de Girona \\ Girona, España
}

\author{
${ }^{2}$ Dept. Ciencias de la Educación, Lenguaje, \\ Cultura y Artes, Ciencias Historico-Jurídicas y \\ Humanísticas y Lenguas Modernas \\ Universidad Rey Juan Carlos \\ Madrid, España
}

\author{
${ }^{2}$ Dept. Història \\ i Arqueologia \\ Universitat de Barcelona \\ Barcelona, España
}

\begin{abstract}
Resumen- En el marco de la asignatura del grado de Arqueología de la Universidad de Barcelona se plantean una serie de actividades prácticas para dotar al alumnado de una serie de técnicas y conocimientos que puedan aplicar en futuros trabajos e investigaciones. Fue Antonio Aguilera, uno de los desarrolladores originales de la base de datos de epigrafía anfórica del CEIPAC quien fomentó su uso como parte de una actividad hipotética donde el alumnado universitario se enfrentaba al hallazgo de una inscripción sobre cerámica en una excavación arqueológica. Esto les permitiría conocer el manejo del corpus digital esencial para leer y comprender los textos epigráficos latinos donde hallar paralelos relativos a la inscripción. Esta concomitancia les permitiría poder datar los estratos arqueológicos, conocer el tipo de producto que llega a la excavación y cuál fue la distribución de los contenedores alimenticios. Aquí la práctica se traslada al portal Roman Open Data (https://romanopendata.eu), una interfaz exploratoria desarrollada en el marco del proyecto europeo EPNet que permite geolocalizar la búsquedas epigráficas sobre un mapa, consultando mediante el empleo de herramientas computacionales poder analizar los datos epigráficos sobre ánforas a los miembros de la comunidad académica, a la vez que se promueven los principios y prácticas de la Ciencia Abierta en el contexto de las Humanidades Digitales.
\end{abstract}

Palabras clave: formación, arqueología, epigrafía, historia antigua, software, humanidades digitales, ciencia abierta.

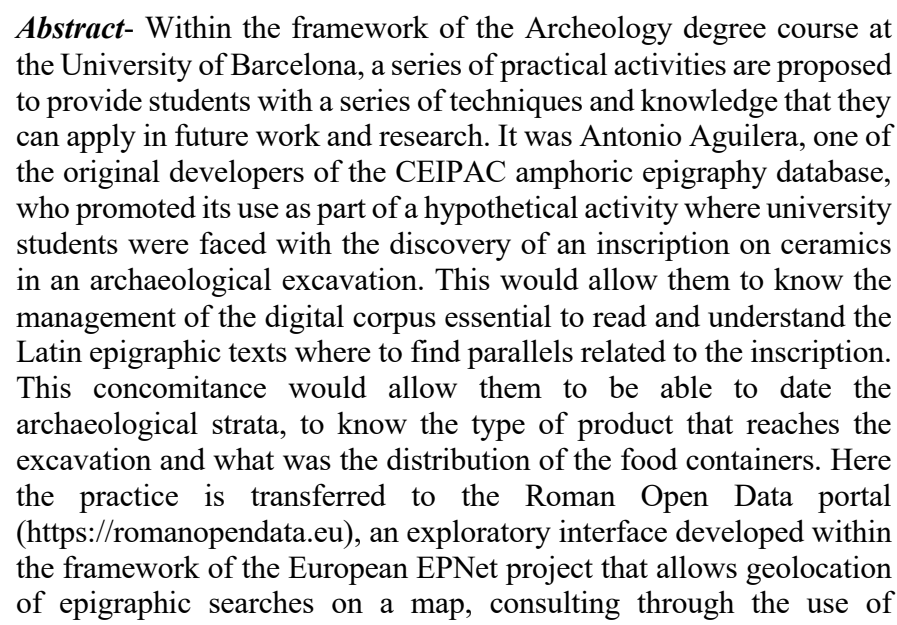

computational tools to be able to analyze epigraphic data on amphorae to members of the academic community, while promoting the principles and practices of Open Science in the context of Digital Humanities.

Keywords: training, archeology, epigraphy, ancient history, software, digital humanities, open science.

\section{INTRODUCCIÓN}

La asignatura de Epigrafia del grado de Arqueología de la Universidad de Barcelona consta de un alto contenido práctico y entre sus objetivos principales destaca el deseo de dotar a los alumnos de los conocimientos y técnicas esenciales para leer y comprender los textos epigráficos latinos. Esta concomitancia les permitiría poder datar los estratos arqueológicos, conocer el tipo de producto que llega a la excavación y cuál fue la distribución de los contenedores alimenticios. Entre los diversos trabajos desarrollados durante el curso, se propone al alumnado realizar una práctica con una de las formas de epigrafía más común en las excavaciones arqueológicas, la hallada sobre cerámica. Esta epigrafía tiene que ver con las diversas fases de la trazabilidad de la cerámica y en el caso particular de las ánforas se divide entre marcas ante cocturam (hechas antes de la cocción de la pieza), donde destacan los grafitos y los sellos (en relación a los sellos y significado, véase Remesal 1986 y Moros 2019; y sobre los grafitos Rodríguez 1993 y Ozcáriz et al. 2020), y las marcas post cocturam (hechas después de la cocción de la pieza), entre las que se encuentras los grafitos y las marcas pintadas conocidas como tituli picti (en relación a los tituli picti y significado, véase Aguilera 2001; 2007 y Remesal, Aguilera 2014).

El caso de estudio que se plantea a los estudiantes nace del hallazgo de alguna de estas inscripciones en una hipotética excavación y como, gracias al empleo de la base de datos de epigrafía anfórica del CEIPAC pueden hallar paralelos relativos a la inscripción analizada. La base de datos de epigrafía anfórica latina del CEIPAC consta de más de 50.000 registros y está próxima a los dos millones de datos (http://ceipac.ub.edu). Gracias a la reunión virtual de un gran número de catálogos el investigador dispone de una gran masa de información reducida 
en un solo punto donde organizar la exploración de epigrafía anfórica (Remesal et al. 2000, 2008 y 2015a; Remesal 2012; Aguilera, Berni 2001; Aguilera 2004; Pérez 2014).

Si tomamos de referencia uno de los recipientes cerámicos mejor conocidos por su prolífica producción durante los tres primeros siglos d.C. a lo largo de los ríos Genil y Guadalquivir, en la actual Andalucía, el ánfora olearia del tipo Dressel 20 (Remesal 2018), podemos detallar gracias al estudio epigráfico su lugar de producción específico, así como la cronología de fabricación y embarque. En una primera fase, en los grafitos previos a la cocción del envase hallaríamos representado al propietario de la alfarería o al artesano especializado en la fabricación del envase, a continuación el sello mostraría mediante un mensaje abreviado al dueño de la alfarería, al productor del contenedor o al dueño del aceite envasado, y por último los tituli picti anunciarían la tara del envase, el peso en libras romanas del aceite envasado, el lugar del control fiscal ejercido y el año consular del envase y los agentes privados dueños del aceite y encargados de su distribución al amparo del Estado romano (Fig. 1).

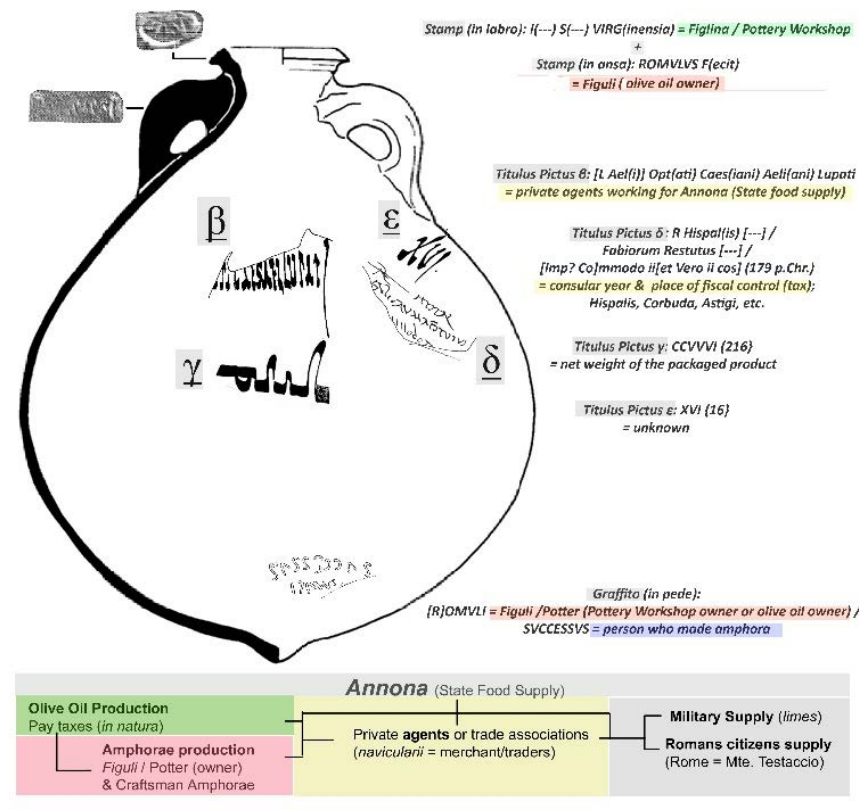

Figura 1 Distribución epigráfica sobre ánfora oleario del tipo Dressel 20.

\section{CONTEXTO}

A continuación, se proponen al alumnado diferentes casos epigráficos para iniciar la práctica. El ejercicio consiste en ofrecer a los alumnos una fotografía del epígrafe y guiarlos en la búsqueda de otros ejemplos entre la maraña de datos de la base epigráfica del CEIPAC. Estas actividades docentes forman parte del último escalón diseñado por el grupo CEIPAC, con unas primeras fases dedicadas a la acumulación de datos y su análisis, y que en la actualidad dedica parte de su tiempo a la transferencia docente y social de sus investigaciones. Así, el objetivo de la presente comunicación es guionizar cuales son los pasos que deben seguir los alumnos en el empleo del corpus digital del CEIPAC y su futura aplicabilidad en hallazgos reales de estos materiales.

En esta línea, es normal que al inicio del curso de Epigrafía la base de datos tenga un incremento en las visitas originado por la suma de 30 o 40 nuevos usuarios. Así, tomando de ejemplo los años 2018 y 2019, y capturando su uso mediante una visualización vemos un incremento de las visitas a partir de los meses de septiembre a octubre, hecho derivable en gran medida a la incorporación de los usuarios procedentes de los cursos de Epigrafía.

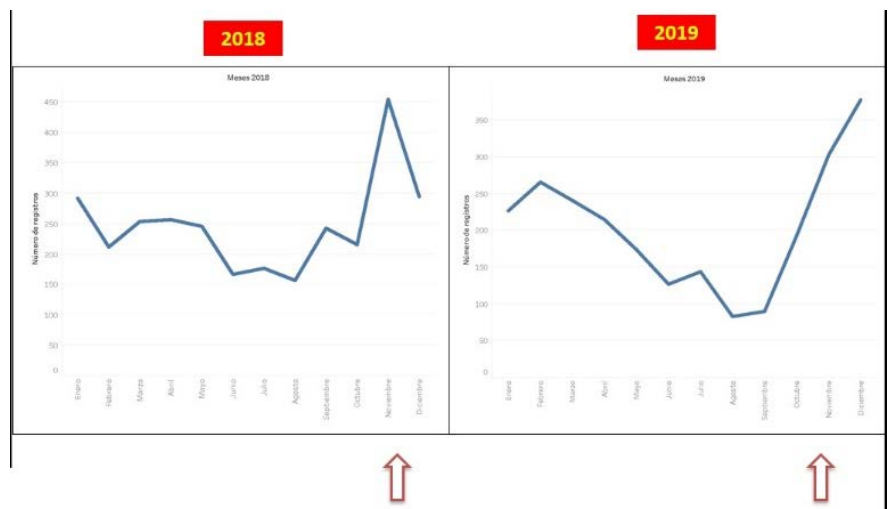

Figura 2. Frecuencia de uso mensual de la base de datos del CEIPAC durante los años 2018 y 2019.

Para poder disponer de esta información internamente desde el CEIPAC se pide al usuario que se registre en la base. Una vez solicitado el acceso a la base de datos, se dan a conocer las últimas publicaciones vaciadas en la base, así como de las últimas incorporaciones en la base de datos bibliográfica. Por otro lado, se propone a partir de este momento citar las fichas epigráficas mediante la referencia del número CEIPAC. En paralelo, una visita al navegador geográfico nos permite hacernos una idea de la magnitud de los datos vaciados; geolocalizando los sellos, grafitos y tituli picti en los diferentes países modernos, ciudades y otras localizaciones específicas. En este línea, la herramienta de búsqueda epigráfica también permite realizar búsquedas según su tipología, ya sea de forma general o detallada, pudiendo combinar diversos campos en la misma query complejizándola para obtener resultados más específicos.

A continuación, se propone al alumnado diversos casos epigráficos que podrían hallar en una excavación ficticia o en una revisión de materiales inédita y cuya búsqueda podrán realizar en la tradicional base de datos del CEIPAC o en su extensión exploratoria Roman Open Data.

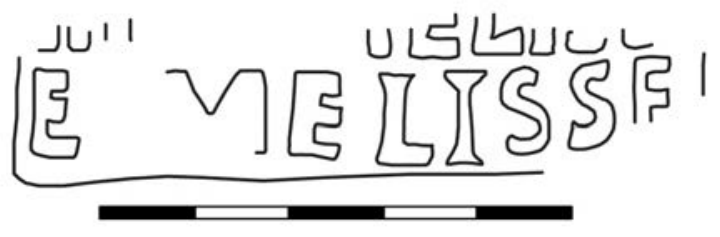

Figura 3. Propuestas epigráficas de estudio: Sello II[IVNIM]ELISS[I]/E[T]MELISSE (CEIPAC 41498).

Pongamos que el alumno se encuentra un sello de dos líneas y cuyo estado de conservación solo permite leer las letras completas ...e.. elisse. Si, por ejemplo, realizásemos la búsqueda del epígrafe propuesto en la base de datos con el siguiente texto ' $\% \mathrm{e} \%$ elisee' obtendríamos más de 240 resultados del sello hallado en Las Delícias (Écija, Sevilla) con el desarrollo completo IIIVNIMELISSEETMELISSE; una producción conjunta de ánforas de un tal Iunius Melissus junto 
a Iunia Melissa (Berni 2008) Estos resultados podrán visualizarse en formato de texto o mediante imágenes. A continuación, podrán consultarse la ficha detallada de cada epígrafe, permitiendo conocer el desarrollo de todos sus campos, desde el lugar de conservación, al tipo anfórico, pasando por su datación y otros campos reseñables como la literatura, donde destaca la cadena bibliográfica vinculada a su publicación (Fig 4). Cabe destacar la opción añadida en la que se permite al usuario notificar errores y descargar la información de la ficha para su uso particular.

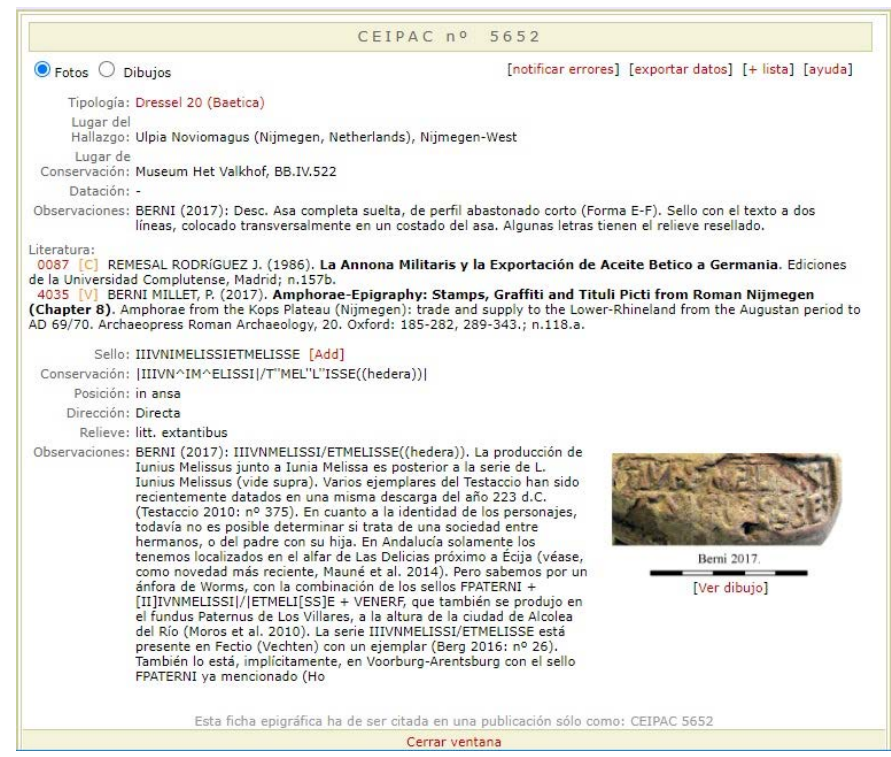

Figura 4. Ficha epigráfica resultante de la búsqueda del sello IIIVNIMELISSI/ETMELISSE en la base CEIPAC (5652).

\section{DESCRIPCIÓN}

Recientemente, en el marco del proyecto europeo EPNet: Production and distribution of food during the Roman Empire: Economics and Political Dynamics (ERC-2013-ADG 340828, Remesal et al. 2015b) se ha desarrollado el portal Roman Open Data (https://romanopendata.eu), la culminación de los esfuerzos de representación del conocimiento acumulado durante décadas de los proyectos del CEIPAC (sobre su uso véase Palacín et al. 2017 y 2020; Gimenez et al. 2018. Véanse tutoriales de la herramienta en Pérez 2020a y 2020b). El objetivo del portal es permitir a los historiadores mediante el empleo de herramientas computacionales poder analizar los datos epigráficos sobre ánforas a los miembros de la comunidad científica, a la vez que se promueven los principios y prácticas de la Ciencia Abierta en el contexto de las Humanidades Digitales. El sistema de información empleado se basa en un motor de integración y acceso a datos basado en ontologías, llamado Ontop (Calvanese et al. 2015 y 2016; Mosca et al. 2015). La herramienta permite geolocalizar la respuesta sobre un mapa, pudiendo concretar más a través de las vistas de Tabla o de Galería, visualizando la información de sus fichas. La interfaz principal es un gráfico de conocimiento virtual, que se presenta en RDF, se ajusta a la ontología desarrollada y que puede consultarse a través de un punto final SPARQL. Asimismo, uno de los éxitos de la interfaz radica en su fácil empleo, permitiendo a un usuario sin grandes conocimientos técnicos explorar los datos sin tener que preocuparse por escribir estas consultas en lo que nos gusta llamar, lenguaje de los ordenadores.

A continuación, si realizásemos una consulta sobre el epígrafe IIIVNIMELISSIETMELISSE, señalando el texto concreto a visualizar, en este caso ...elisse obtendríamos el siguiente resultado:

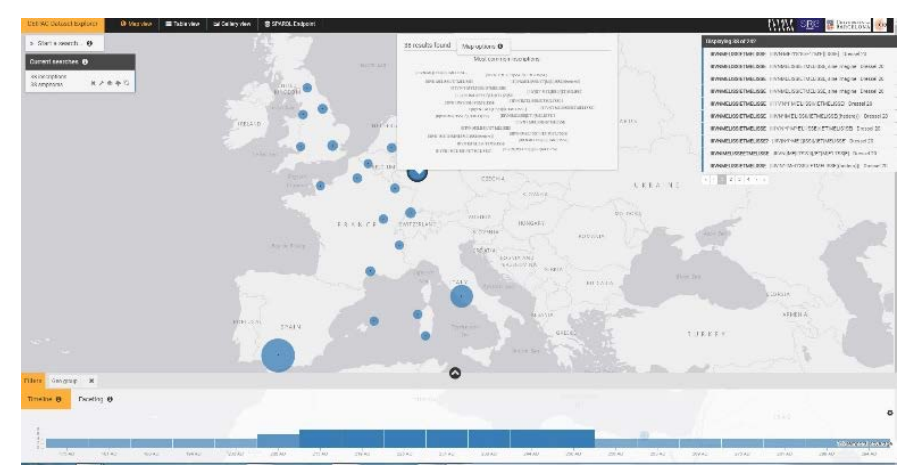

Figura 5. Búsqueda del texto ...elissi en Roma Open Data (del sello II Ivni Melissi et Melisse).

Roman Open Data ofrece múltiples posibilidades de búsqueda epigráfica, tipológica e incluso bibliográfica. Poder enlazar querys permite ofrecer resultados más complejos en cuestión de segundos, agilizando el análisis de los datos como nunca habíamos conocido. A la consulta propuesta se pueden concatenar de otras, ya sean del tipo epigráfico, tipológico o bibliográfico. Estos resultados aparecerán expuestos sobre la misma visualización, comparando los diversas consultas y con la posibilidad de filtrar sobre cualquiera de los nodos. Igualmente, estas sumas siempre podrán ocultarse con una fácil pulsación sobre un icono en forma de ojo $\$$. En paralelo, siempre se ofrece la posibilidad de una consulta más detallada mediante el sistema clásico de fichas en la en la sección de Tablas y Galería. Y como hemos comentado, sumándonos al movimiento de Ciencia Abierta, se permite la descarga de los datos consultados en un formato .csv o .xls, ya sean en su totalidad o filtrados. Para ello basta con clicar sobre el icono en forma de nube con una flecha $\$$. Otra opción permite descargar la visualización resultante mediante una captura de pantalla, ofreciendo varias opciones sobre los resultados a mostrar en el mapa, ya sea a través de nodos, mapas de calor, o con la base de un mapa moderno o uno del Imperio Romano. Además, se permite al usuario seleccionar una franja temporal conocida en cada una de las producciones epigráficas, reduciendo el campo de búsqueda e incluso mediante el uso de la herramienta Faceting se pueden definir consultas más concretas o proponer nuevos campos de búsqueda.

A modo de ejemplo, podemos comparar la distribución de la producción de los Melissii con otra producción del siglo III d.C. que aparece codificada bajo el texto PNN. En la siguiente consulta además de observar los resultados generales de la búsqueda del sello PNN, la que supera los 500 ejemplares, hemos añadido una tercera consulta más detallada de un sello de la misma lectura con el fin de observar la potencialidad de la herramienta: filtrando por su hallazgo en asentamientos civiles de Italia, de lectura directa, excisos y sobre las asas del ánfora. En esta línea, los resultados temporales se pueden unificar bajo un mismo marco cronológico para compararlos mejor. 


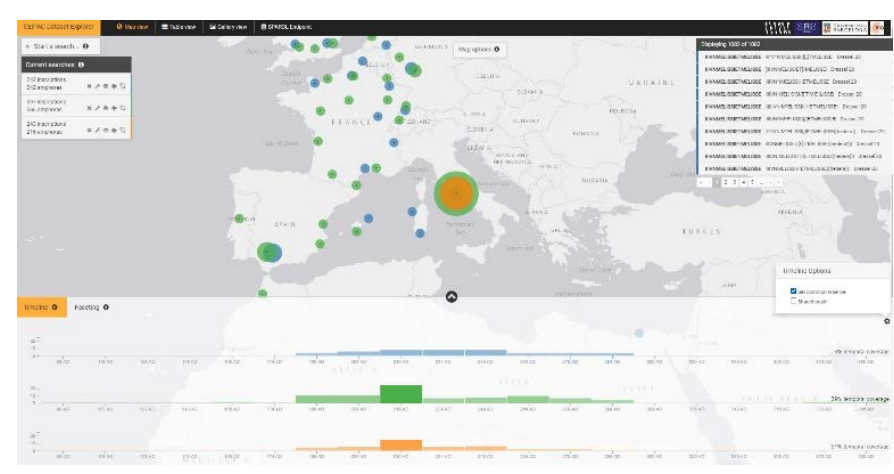

Fig. 6. Búsqueda del texto ...elissi en Roma Open Data (del sello II Ivni Melissi et Melisse) -en azul. En verde los resultados de la búsqueda del texto PNN. En naranja los resultados del texto PNN filtrado en una búsqueda avanzada.

\section{Resultados}

Supongamos que la propuesta de trabajo al alumnado sobre el hallazgo de epigrafia anfórica en una excavación hipotética acaba por materializarse una investigación real y que nuestros alumnos se encuentran ante el hallazgo de un asa de ánfora con el ejemplar arriba analizado. Si bien este caso ya se nos ha planteado de forma interna en el seno del grupo CEIPAC por investigadores que solicitan soporte para descifrar el significado de la epigrafía que están estudiando y a los que también se les deriva a emplear la herramienta diseñada, en este caso, ¿qué información obtendrían de su consulta en Roman Open Data?

Rápidamente observarán mediante la comparativa de las imágenes que están frente a un ejemplar incompleto del sello IIIVNMELISSIETMELISSE. Además, el 100\% de la muestra conocida en el corpus digital del CEIPAC con más de 240 registros pertenecen solo a esta lectura. Verían que se trata de una producción conocida en hasta 53 lugares distintos y cuyo lugar de producción se asoció al alfar de Las Delicias, próximo a Écija en la actual Andalucía (Mauné et al. 2014). Indirectamente, conocerían el tipo anfórico al que se asoció, las ánforas olearias del tipo Dressel 20 producidas en la provincia romana de la Baetica; lo que les permitiría conocer el producto contenido. Con una consulta a los ejemplares de la misma lectura obtendrían un listado de la bibliografía básica y especializada del epígrafe, donde hallar las posibles interpretaciones sobre la identidad de los personajes vinculados a la marca, en este caso, quizás una sociedad entre los hermanos Iunius Melissus junto a Iunia Melissa, o la del padre con su hija (Remesal 1986; Berni 2008 y 2017). También sabrían que este sello se combinó a otros dos sellos, el FPATERNI y el VENERF, por su hallazgo en otra ánfora de Worms y Wiesbaden, actual Alemania (CEIPAC 11 y 14588 respectivamente), también producido en el fundus Paternus de Los Villares, a la altura de la ciudad de Alcolea del Río (Moros et al. 2010). Y gracias a las excavaciones en el Monte Testaccio (Roma) (Remesal 2019) podrán inferir una datación del siglo III d.C., donde otros ejemplares de la misma lectura han sido datados en una misma descarga del año 223 d.C., en especial en el estrato C 50-70 (Remesal 2010, n. 375, 375a1 y 375a2; CEIPAC 28721-28723), donde el ejemplar n. 375a2 ha aparecido junto a un titulus pictus con datación consular $[R$ astigis $]$ arca p(endo) ccxl/[---]+ et euthyches/[ma]ximo ii $[$ et $]$ aeliano cos (Remesal, Aguilera 2010, n. 81; CEIPAC 28977)
Por último, gracias a la representación gráfica sobre el mapa podría observar su distribución por el Mediterráneo occidental, con gran presencia en Roma y en la frontera renana.

\section{CONCLUSIONES}

Para finalizar, el ejercicio propuesto dota a los alumnos de las herramientas necesarias para poder conocer de forma rápida y detallada la información que se encuentra detrás de los mensajes epigráficos, algunos codificados y ampliamente representados y estudiados por el CEIPAC.

La actividad docente se desarrolla desde el curso 2015/2016 en el grado de Arqueología utilizando el esquema tradicional de la base de datos del CEIPAC. Las clases constan de una media de 15/20 alumnas/os y el trabajo se propone con carácter individual, previa instrucción sobre el funcionamiento de las base de datos. En la totalidad de los casos, los datos solicitados son hallados por el alumnado, prueba del buen uso de la base epigráfica, lo que se valora positivamente en su evaluación. En consecuencia, se dota al alumnado de nuevas herramientas de análisis en el marco de las Humanidades Digitales con una Epigrafía inmersa en plena era digital, potenciando así procesos que agilicen futuros estudios, capaces de abordar grandes conjuntos de datos. Por nuestra parte desconocemos si los organizadores de contenidos epigráficos de otras bases de datos como la Epigraphic Database Heidelberg (https://edhwww.adw.uni-heidelberg.de), la Epigraphic-Datenbank Clauss-Slaby (http://www.manfredclauss.de) o la Epigraphic Database Rome (http://www.edr-edr.it) promueven su difusión y empleo entre el alumnado universitario.

Esto permite conocer, gracias al vinculo tipológico de la pieza asociado al epígrafe, el producto fabricado, comercializado y/o consumido en el lugar de hallazgo cerámico. En esta línea, si los lugares de producción del producto son conocidos se pueden elaborar rutas comerciales para el consumo de estos productos y último, a partir de las dataciones contextuales o tipológicas pueden inferir un marco temporal para el epígrafe analizado y por lo tanto proponer cronologías para la zona del sondeo arqueológico del cual procede la pieza, muchas de ellas tan precisas como la fecha de un año consular.

\section{AgRAdeCIMIENTOS}

Agradecemos la financiación de la investigación a los siguientes organismos: JPG financiado por Juan de la CiervaFormación-2019 (Agencia Estatal de Investigación (Ministerio de Ciencia e Innovación) REF: FJC2019-040688-I, JMBL por Ariadne+ y AAM por los proyectos HAR2017-85635-P, 2017 SGR 512.

\section{REFERENCIAS}

AGUILERA, A. (2001). "Los tituli picti delta del convento astigitano en el primer tercio del s.III d.C." en Congreso Internacional Ex Baetica Amphorae. Conservas, aceite y vino de la Bética en el Imperio Romano (Écija y Sevilla, 17 al 20 de Diciembre de 1998). Écija: Gráficas Sol.

AGUILERA, A. (2002). El monte Testaccio y la llanura subaventina. Topografía extra portam Trigeminam. Roma: Consejo Superior de Investigaciones Científicas.

AGUILERA, A. (2004). "Sistematización de los tituli picti anfóricos para la base de datos CEIPAC" en Remesal Rodríguez, J. (Ed.) Epigrafía anfórica (Col·lecció 
Instrumenta 17). 105-126. Barcelona: Publicacions i Edicions de la Universitat de Barcelona.

AGUILERA, A. (2007). "Evolución de los tituli picti delta de las ánforas Dressel 20 entre mediados del siglo I y mediados del siglo III" en Mayer Olivé, M., Baratta, G., Guzmán Almagro, A. (Eds.) Acta XII Congressus Internationalis Epigraphiae Graecae et Latinae (Barcelona, 3-8 Septembris 2002) (Monografies de la Secció Històrico-Arqueològica X). Barcelona: Institut d'Estudis Catalans.

AGUILERA, A. y BERNI, P. (2001). "Las bases de datos y las Ciencias de la Antigüedad" en Arqueo Mediterrània, 7, 57-63.

BERNI, P. (2008). Epigrafía anfórica de la Bética. Nuevas formas de análisis (Col·lecció Instrumenta 29). Barcelona: Publicacions i Edicions de la Universitat de Barcelona.

BERNI, P. (2017). “Amphorae-Epigraphy: Stamps, Graffiti and Tituli Picti from Roman Nijmegen (Chapter 8)" en Amphorae from the Kops Plateau (Nijmegen): trade and supply to the Lower-Rhineland from the Augustan period to AD 69/70 (Archaeopress Roman Archaeology 20). 185-282, 289-343. Oxford: Archaeopress.

CALVANESE et al. (2015) = CALVANESE, D., MOSCA, A., REMESAL, J., REZK, M. y RULL, G. (2015) “A 'Historical Case' of Ontology-Based data Access" en 2015 Digital Heritage. 291-298. Granada.

$<$ https://doi.org/10.1109/DigitalHeritage.2015.7419510>

[Consulta: 27 de marzo de 2021].

CALVANESE et al. (2016) = CALVANESE, D., LIUZZO, P., MOSCA, A., REMESAL, J., REZK, M. y RULL, G. (2016). "Ontology-based data integration in EPNet: Production and distribution of food during the Roman Empire" en Engineering Applications of Artificial Intelligence, 51, 212-229.

$<$ https://doi.org/10.1016/j.engappai.2016.01.005> [Consulta: 27 de marzo de 2021].

GIMENEZ et al. (2018) = GIMENEZ, X., RULL, G., PÉREZ, J., MOSCA, A., RONDELLI, B. y REMESAL, J. (2018). "Roman Open Data". Open Science \& The Humanities Conference. Poster. Barcelona: Universitat de Barcelona.

$<$ http://hdl.handle.net/2445/147002 $>$ [Consulta: 27 de marzo de 2021].

MAUNÉ et al. (2014) = MAUNÉ, S., GARCÍA, E., BOURGEON, O., CORBEEL, S., CARRATO, CH., GARCÍA, S., BIGOT, F. y VÁZQUEZ, J. (2014). "L'atelier d'amphores à huile Dr. 20 de Las Delicias à Ecije (Prov. de Séville, Espagne)" en Société française d'étude de la céramique antique en Gaule (SFECAG). Actes du congrès de Chartres, 29 mai-1er juin 2014. Entre Seine et Loire: les Carnutes. Des faciès céramiques contrastés. Actualité des recherches céramiques, 419-444.

$<$ https://halshs.archives-ouvertes.fr/halshs-02129872>

[Consulta: 27 de marzo de 2021].

MOROS DÍAZ, J. (2019) Análisis epigráfico de los sellos olearios béticos hallados en centros de producción: el caso de la zona productora de la Scalensia. Tesis doctoral. Barcelona: Universitat de Barcelona.

$<$ http://hdl.handle.net/2445/133685> [Consulta: 27 de marzo de 2021].

MOROS et al. (2010) = MOROS, J., BAREA, J.S., BAREA, J.L. y SOLÍS, J. (2010). "Propiedades de los severos en la Bética: la figlina Paterna” en Blázquez Martínez, J.M. y Remesal Rodríguez, J. (Eds.) Estudios sobre el Monte Testaccio (Roma) V (Col·lecció Instrumenta 35). Barcelona: Publicacions i Edicions de la Universitat de Barcelona.

MOSCA et al. (2015) = MOSCA, A., REMESAL, J., REZK, M. y RULL, G. (2015). "Knowledge Representation in EPNet" en Morzy, T., Valduriez, P. y Bellatreche, L. (Eds.) New Trends in Databases and Information Systems. ADBIS 2015. Communications in Computer and Information Science, vol. 539. 427-437. Cham: Springer International Publishing.

$<$ https://doi.org/10.1007/978-3-319-23201-0_43> [Consulta: 27 de marzo de 2021].

OZCÁRIZ et al. (2020) = OZCÁRIZ, P., PÉREZ, J. y HEREDERO, J. (2020). "The Logistics of Marking in the Baetic Amphoras. The Use of Numerals in the Organizational Systems of Ceramic Productions" en Studia Antiqua et Archaeologica, 26(2), 231-247.

$<$ http://saa.uaic.ro/articles/SAA.26.2.2020.231-247.pdf> [Consulta: 27 de marzo de 2021].

PALACÍN et al. (2017) = PALACÍN, C., PÉREZ, J. y REMESAL, J. (2017). "Amphoric epigraphy and Roman Open Data: Laietanian wine amphorae as a case study". European Meeting on Ancient Ceramics (EMAC), 15th edition. Universitat de Barcelona (16-18 de setembre de 2017). Poster. Barcelona: Universitat de Barcelona

$<$ http://hdl.handle.net/2445/146998> [Consulta: 27 de marzo de 2021].

PALACÍN et al. (2020) = PALACÍN, C., PÉREZ, J. y RULL, G. (2020). "Epigrafia amfòrica i roman open data: Les àmfores del litoral central de Catalunya com a cas d'estudi" en Laietania, 21, 97-132.

PÉREZ, J. (2014). "La base de datos on line del Ceipac. Los tituli picti” en Ar@cne, 190. Universidad de Barcelona, Barcelona.

<http://http://www.ub.edu/geocrit/aracne/aracne-190.htm> [Consulta: 27 de marzo de 2021].

PÉREZ, J. (2020a). "La epigrafía anfórica. De la ficha al Banco de Datos digital del CEIPAC y el portal RomanOpenData”. Youtube $<$ https://youtu.be/Cwt3X1O02rI > [Consulta: 28 de marzo de 2021]

PÉREZ, J. (2020b). "Roman Open Data: computational tool to analyze the Roman Empire trading system". Youtube $<$ https://youtu.be/Xpo612g21SA $>$ [Consulta: 28 de marzo de 2021]

REMESAL, J. (1986). La Annona Militaris y la Exportación de Aceite Betico a Germania. Madrid: Ediciones de la Universidad Complutense. 
REMESAL, J. (2010). "Los sellos" en Blázquez, J.M. y Remesal, J. (Eds.) Estudios sobre el monte Testaccio: Roma V (Col·lecció Instrumenta 35). Barcelona: Publicacions i Edicions de la Universitat de Barcelona.

REMESAL, J. (2019) "Monte Testaccio (Rome, Italy)" en Smith, C. (Ed.) Encyclopedia of Global Archaeology.

$<$ https://doi.org/10.1007/978-3-319-51726-1 3331-1> [Consulta: 27 de marzo de 2021].

REMESAL, J. (2012) "Corpus versus Catalog, propuestas sobre una vieja cuestión” en Fuchs, M.E., Sylvestre, R. y Heidenreich, C.S. (Eds.) Inscriptions mineures: nouveautes et reflexions. Actes du premier colloque Ductus (19-20 juin 2008, Université de Lausanne). 83-93. Bern, Berlin, Bruxelles, Frankfurt am Main, New York, Oxford, Wien.

REMESAL, J. (2018). "Las ánforas olearias béticas Dressel 20" en Remesal, J. (Ed.) Colonia Ulpia Traiana (Xanten) y el Mediterráneo. El comercio de alimentos (Col-lecció Instrumenta 63). 275-420. Barcelona: Publicacions i Edicions de la Universitat de Barcelona.

REMESAL, J. y AGUILERA, A. (2010). "Los tituli picti” en Blázquez, J.M. y Remesal, J. (Eds.) Estudios sobre el monte Testaccio: Roma V. (Col·lecció Instrumenta 35). Barcelona: Publicacions i Edicions de la Universitat de Barcelona.

REMESAL, J. y AGUILERA, A. (2014). "Los tituli picti" en Blázquez, J.M. y Remesal, J. (Eds.) Estudios sobre el monte Testaccio: Roma VI (Col·lecció Instrumenta 47). 39-414. Barcelona: Publicacions i Edicions de la Universitat de Barcelona.

REMESAL et al. (2000) = REMESAL, J., BERNI, P. y AGUILERA, A. (2000). "Internet. Evaluador y difusor de la ciencia histórica" en Oliveira, J.V. (Ed.) $3^{\circ}$ Congresso de Arqueología Peninsular. Utad, Vila Real, Portugal,
Setembro de 1999, Vol. 9. Contributos das ciencias e das tecnologias para a arqueologia da Península Ibérica. 475488. Porto.

REMESAL et al. (2008) = REMESAL, J., BERNI, P. y AGUILERA, A. (2008). "Amphoreninschriften und ihre elektronische Bearbeitung" en Hainzmann, M. y Wedenig, R. (Eds.) Instrumenta Inscripta Latina II. Akten des 2. Internationalen Kolloquiums. Klagenfurt, 5.-8. Mai 2005. 247-264. Klagenfurt.

REMESAL et al. (2015a) = REMESAL, J., AGUILERA, A., GARCÍA, M., MARTÍN-ARROYO, D.J., PÉREZ, J. y REVILLA, V. (2015). "Centro para el Estudio de la Interdependencia Provincial en la Antigüedad Clásica (CEIPAC)" en Pyrenae: revista de prehistòria $\mathrm{i}$ antiguitat de la Mediterrània Occidental, 46: 1, 245-275.

$<$ https://www.raco.cat/index.php/Pyrenae/article/view/304898 $>$ [Consulta: 27 de marzo de 2021].

REMESAL et al. (2015b) = REMESAL, J., DÍAZ-GUILERA, A., RONDELLI, B., RUBIO, X., AGUILERA, A., MARTÍN-ARROYO, D.J., MOSCA, A. y RULL, G. (2015). "The EPNet Project. Production and distribution of food during the Roman Empire: Economics and Political Dynamics" en Orlandi, S., Santucci, R., Casarosa, V. y Liuzzo, P.M. (Eds.) Information Technologies for Epigraphy and Cultural Heritage Proceedings of the First EAGLE International Conference. 455-464. Roma: EUROPEANA Eagle Project.

$<$ http://hdl.handle.net/2117/85564> [Consulta: 27 de marzo de 2021].

RODRÍGUEZ, E. (1993). "Graffiti e produzione anforaria della Betica" en Harris, W.V. (Ed.) The Inscribed Economy (Ann Arbor 1993), 95-107. 